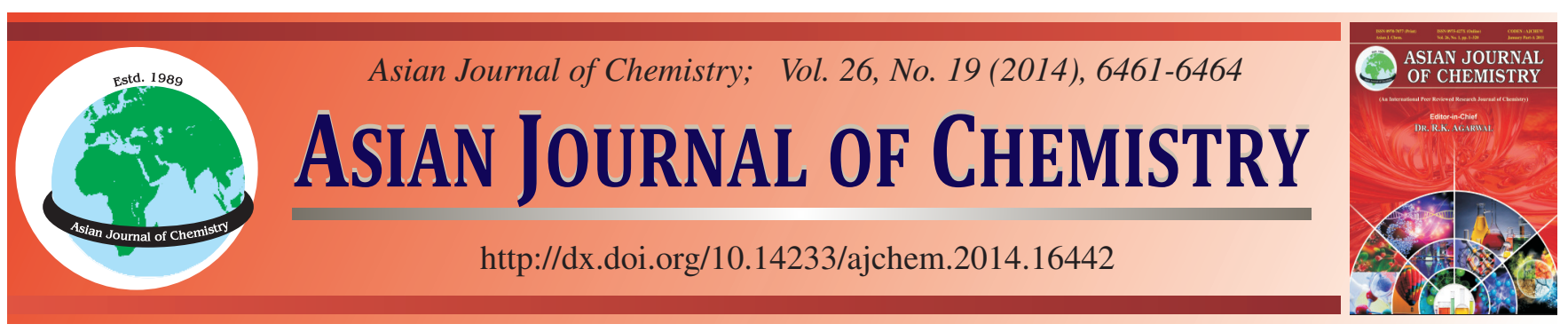

\title{
Selective Adsorption Behaviour of Mesoporous Silica SBA-15-N-Acetylcysteine Imprinted Polymer Based on Surface Molecular Imprinting Technique
}

\author{
XiaOlan Zhu ${ }^{1, *}$, Qingsheng ZhU ${ }^{2}$, Jun YAnG $^{1}$ and Yun GaO ${ }^{1}$
}

${ }^{1}$ Research Center of Tobacco and Health, University of Science and Technology of China, Hefei 230052, P.R. China

${ }^{2}$ Technique Center of Modern Education, Anhui Jianzhu University, Hefei 230022, P.R. China

*Corresponding author: Fax: +86 551 63492065; Tel: +86 551 63492060; E-mail: zx18906@ustc.edu.cn

Received: 28 September 2013; $\quad$ Accepted: 16 January 2014; $\quad$ Published online: 16 September 2014;

AJC-15940

\begin{abstract}
A new N-acetylcysteine ion-imprinted polymer (NAC-MIP), which can be used for selective adsorption of N-acetylcysteine from aqueous solutions, was successfully prepared based on the supported material of ordered mesoporous silica SBA-15 with the help of surface molecular imprinting technology. The prepared polymer was characterized by Fourier transmission infrared sepectrometry, X-ray diffraction, scanning electron microscopy and ${ }^{13} \mathrm{C}$ NMR spectroscopy. The results showed that the synthesized polymer possessed highly ordered mesoporous structure. The imprinted silica particle showed high selectivity and fast kinetic binding for the template due to its nanosized wall thickness and high surface area.
\end{abstract}

Keywords: SBA-15, Ordered mesoporous silica, Surface molecular imprinting technology, N-acetylcysteine.

\section{INTRODUCTION}

N-Acetylcysteine, a metabolite of the sulfur-containing amino acid, is a pharmaceutical drug and nutritional supplement used primarily as a mucolytic agent and in the management of paracetamol (acetaminophen) overdose ${ }^{1}$. It is used as a cough medicine because it breaks disulfide bonds in mucus and liquefies them, making it easier to cough up. Acetylcysteine is also sold as a dietary supplement commonly claiming antioxidant and liver protecting effects ${ }^{2}$.

Molecular imprinting can be defined as the assembly of a cross-linked polymer matrix around an imprint molecule that is held in place, either covalently on noncovalently, by judiciously chosen functional monomers ${ }^{3,4}$. Imprinted materials must have binding sites and a specific size and shape. However, the binding sites and imprinted cavities in traditional molecularly imprinted polymers (MIPs) are often embedded so deeply that the substrate molecules cannot easily access to them. This problem can be overcome by surface imprinted polymers ${ }^{5,6}$. An important kind of surface imprinting technique is based on the surface modification of the matrix material. The distribution of the imprinted cavities in the thin polymer layer on matrix material is of great advantage to the fast binding of the template molecules with the recognition sites.

Silica based materials are common supports because they are stable under acidic conditions and no swelling inorganic material and has high mass exchange characteristics. In recent years, silica based mesoporous materials have been universally reported as good solid support due to their stable mesoporous structure, good chemical and mechanical stability and well modified surface properties with abundant $\mathrm{Si}-\mathrm{OH}$ active bonds on the pore walls ${ }^{7,8}$. Commonly, the mesoporous silica can be modified by the post-synthesis or one-pot synthesis. Especially the post-synthesis method does not destroy the mesoporous structural ordering, but functional groups are anchored on the surface or inside the pores of the mesoporous silica. Among the mesoporous silicas, SBA-15 is characterized with highly ordered two-dimensional symmetry possesses hexagonal arrays of uniform pores with ultra large pore diameters, large surface area, high pore volume and thicker pore walls ${ }^{9-11}$. It also shows excellent homogeneity and chemical/mechanical stability. These characteristics of SBA-15 enables itself a potential candidate for inclusion of guest species on the surface.

Herein, we report molecularly imprinted $\mathrm{N}$-acetylcysteine mesoporous silica particles based on a surface molecular imprinting technology modified with SBA-15. To the best of our knowledge, there has been no report focused on surface $\mathrm{N}$-acetylcysteine synthesized by support of SBA-15. The ordered structure of the mesoporous silica was studied by scanning electron microscopy and XRD and the adsorption behaviour of the adsorbents for $\mathrm{N}$-acetylcysteine was investigated using the rebinding tests.

\section{EXPERIMENTAL}

Tetraethylorthosilicate (TEOS), 3-aminopropyltrimethoxysilane (APS), N-acetylcysteine, 3-hydroxypropyl mercapturic 
acid (HPMA), bisphenol A (BPA) and silica (70-230 mesh) were purchased from J\&K Chem-Tech Ltd (Shanghai, China). Triblock copolymer poly(ethylene glycol)-block-poly (propylene glycol)-block-poly(ethylene glycol) (Pluronic $\mathrm{P} 123 ; \mathrm{EO}_{20} \mathrm{PO}_{70} \mathrm{EO}_{20}$ ) was obtained from Aldrich. All other chemicals were of analytical grade and solvents were of HPLC quality. Ultrapure water used for sample preparation was obtained from a MILLI-R04 purification system, (Millipore Corp., Germany).

Synthesis of the NAC-MIP: The hexagonally structured mesoporous silica material was synthesized according to a previously described procedure ${ }^{12}$. Amine-functionalized mesoporous silica was obtained by modification of SBA-15 with $\mathrm{APS}^{13}$. The NAC-imprinted mesoporous silica particles were prepared according to Fig. 1. First, N-acetylcysteine $(0.4 \mathrm{~g})$ was dissolved in $25 \mathrm{~mL}$ of ethanol while stirring. $2 \mathrm{~g}$ of the amine-functionalized mesoporous silica was added and refluxed for $2 \mathrm{~h}$, then $4 \mathrm{~mL}$ TEOS and $3 \mathrm{~mL} 1 \mathrm{~mol} / \mathrm{L} \mathrm{AcOH}$ were added. The mixture was stirred and refluxed for $8 \mathrm{~h}$ and the product was filtered and dried under vacuum at $100{ }^{\circ} \mathrm{C}$ for $12 \mathrm{~h}$. The product was washed twice with ethanol and once with ultrapure water. The remaining solid was extracted by a mixture of ethanol and $6 \mathrm{~mol} / \mathrm{L} \mathrm{HCl}(\mathrm{v} / \mathrm{v}=1: 1)$ in a Soxhlet for $24 \mathrm{~h}$, neutralized with $0.1 \mathrm{~mol} / \mathrm{L}$ patassium hydroxide and washed by utrapure water to remove the molecular template and any other excess polymer. Finally, the powder was dried at $100{ }^{\circ} \mathrm{C}$ for $12 \mathrm{~h}$ under vacuum. The resulting polymer powder (NAC-MIP) was collected using a standard analysis sieve. The non-imprinted functionalized mesoporous silica (NAC-NIP) was prepared using an identical procedure without adding $\mathrm{N}$-acetylcysteine.

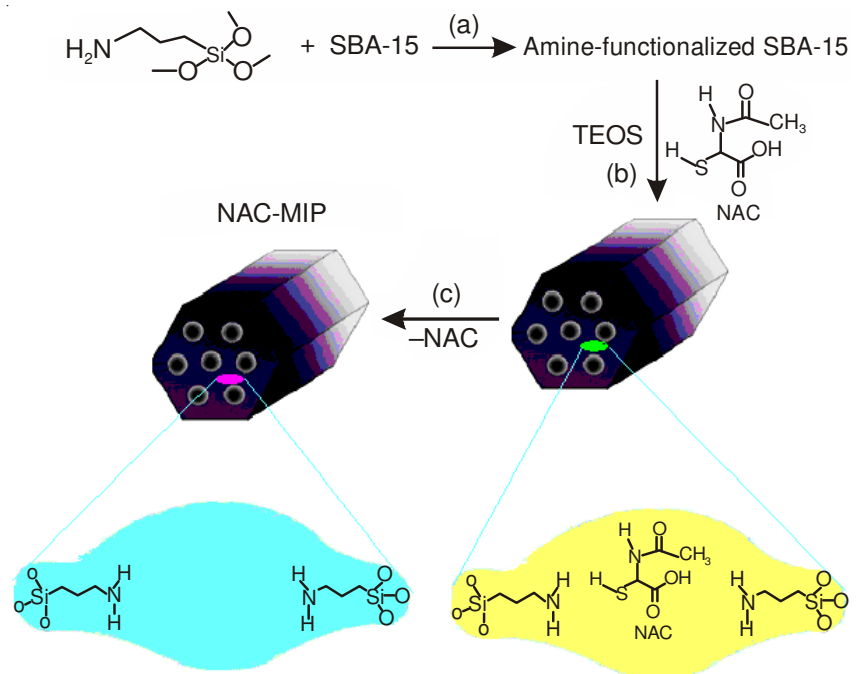

Fig. 1. Synthesis of N-acetylcysteine-MIP: (a) synthesis of the 3-aminopropyl functional mesoporous silica, (b) the process of imprinting and (c) the processs of elution

HPLC analysis: Chromatographic evaluation was performed on an Agilent 1100 series high performance liquid chromatography equipped with 1312 A Binary Gradient Pump, 1313 A Thermostatted Autosampler, G1316 A column oven, G1315 A Diode Array Detector and G1319 A Chemstation. Chromatographic separation was carried out with an Agilent $\mathrm{XDB}-\mathrm{C}_{18}$ column $(250 \mathrm{~mm} \times 4.6 \mathrm{~mm}$ i.d., Particle size $5 \mu \mathrm{m})$.
The mobile phase consisted of $96 \% 20 \mathrm{mM} \mathrm{NaH}_{2} \mathrm{PO}_{4}$ (the $\mathrm{pH}$ was adjusted to 2.5 with $50 \% \mathrm{H}_{3} \mathrm{PO}_{4}$ ) and $4 \%$ acetonitrile at $1 \mathrm{~mL} / \mathrm{min}$. The column temperature was maintained at $35^{\circ} \mathrm{C}$.

Rebinding tests: The washed imprinted mesoporous silica particles (30 mg) (NAC-MIP) and the NAC-NIP were added to aqueous solution of $\mathrm{N}$-acetylcysteine at various concentrations from $20 \mu \mathrm{M}$ to $2.5 \mathrm{mM}$. After incubating for $2 \mathrm{~h}$ at room temperature, the silica particles were isolated by centrifugation. The supernatant was analyzed by HPLC system. The average data of triplicated independent results was used for the analysis. In kinetic test, the SBA-MIP or SBA-NIP particles $(30 \mathrm{mg}$ ) were added to an aqueous solution of $\mathrm{N}$-acetylcysteine (200 $\mu \mathrm{M}, 20 \mathrm{~mL}$ ). Then $0.5 \mathrm{~mL}$ of the mixture was taken at regular time and analyzed after centrifugation.

\section{RESULTS AND DISCUSSION}

To determine the interaction between the recognition site and $\mathrm{N}$-acetylcysteine, we studied the imprinted process using Fourier transform-infrared (FT-IR) spectroscopy (Fig. 2). A peak, which appeared around $1082.22 \mathrm{~cm}^{-1}$, was representative of the Si-O-Si stretching vibrations. Silicon oxide ( $\mathrm{Si}-\mathrm{O}$ ) vibrations were reflected around 801.77 and $464.08 \mathrm{~cm}^{-1}$. The absorptions around 3442.70 and $1631.68 \mathrm{~cm}^{-1}$ resulted from $\mathrm{O}-\mathrm{H}$ vibration, while peaks around $1561.38 \mathrm{~cm}^{-1}$ manifested the $\mathrm{N}-\mathrm{H}$ (primary amine) bending vibration shifted to 1510.64 $\mathrm{cm}^{-1}$. These results indicate that the $-\mathrm{NH}_{2}$ group was successfully grafted onto the surface of mesoporous silica because the $\mathrm{Si}-\mathrm{OH}$ signal was replaced with the $\mathrm{Si}-\mathrm{O}-\mathrm{Si}$ signal and the absorbance at $961.19 \mathrm{~cm}^{-1}$ became sharper ${ }^{14}$.

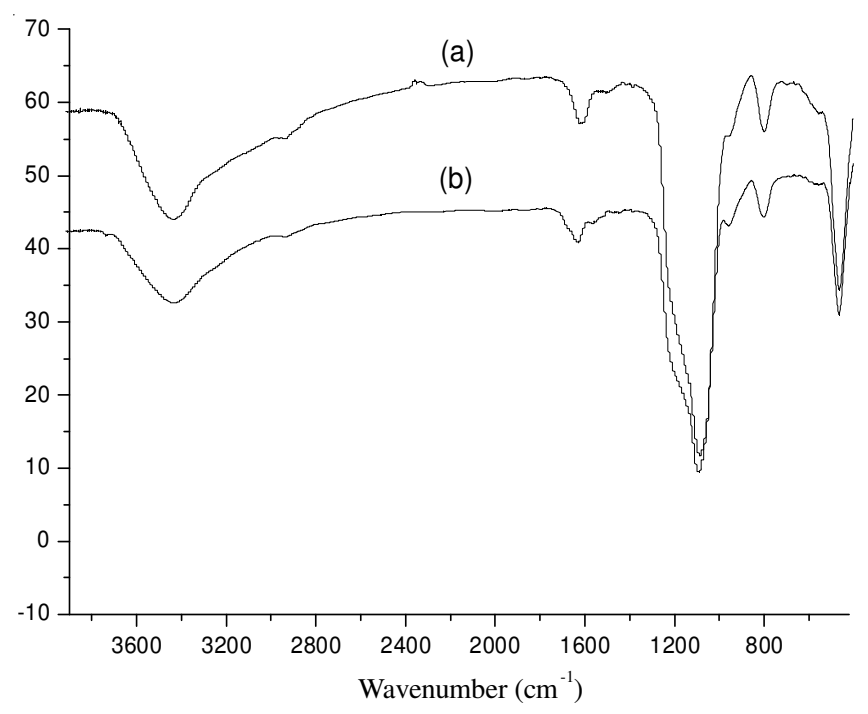

Fig. 2. Fourier transform infrared spectra of the molecularly imprinted mesoporous silica particles: (a) MIP and (b) NIP

To obtain a more comprehensive characterization of the composition of the materials, solid-state nuclear magnetic resonance (SSNMR) spectroscopy was employed. ${ }^{13} \mathrm{C}$ rosspolarized magic-angle spinning (CPMAS) spectra were obtained for all samples to confirm the presence of the expected organic groups. In the MIP-NAC spectrum (Fig. 3a), the -COOH (173.5 $\mathrm{ppm})$ is clearly present, as well as the anchoring propyl groups $(8,22 \text { and } 42 \mathrm{ppm})^{15}$. Residual P123 template (70-80 ppm) is also present. After extraction, these peaks and $-\mathrm{COOH}$ peaks 
(a)

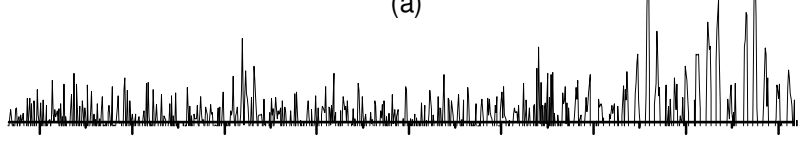

(b)

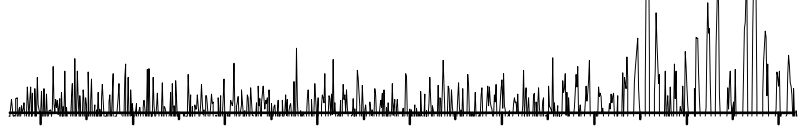

(c)

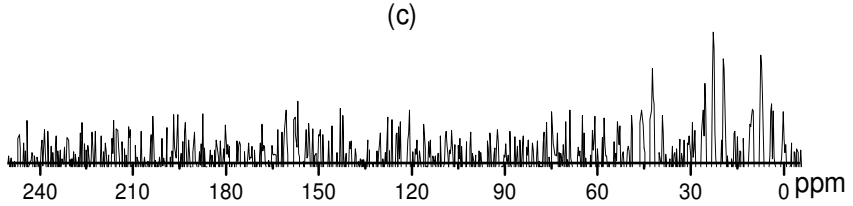

Fig. 3. Solid-state ${ }^{13} \mathrm{C}$ NMR spectra of the mesoporous silica particles: (a) MIP before, (b) MIP and (c) NIP after the extraction of Nacetylcysteine

are eliminated, while the relative intensity of the carbon signals in the remaining $\mathrm{Si}-\mathrm{O}$ groups is unchanged, indicating that most of the imprint molecules were removed without affecting the anchoring groups.

Effect of organic groups on pore structure: The preservation of the mesoporous structure was confirmed visually by scanning electron microscopy (SEM) of NAC-MIP, NAC-NIP and SBA-15. As shown in Fig. 4, NAC-MIP and NAC-NIP showed relatively regular fibre stick and some evidence of a hexagonal pore structure, but the pores in NAC-MIP and NACNIP were significantly more uneven and disordered than those in SBA-15. This trend was also evident in the small-angel X-ray diffraction (XRD) data obtained for each of the samples. The broader reflection was obtained for NAC-MIP and that of NACNIP was slightly sharper (Shown in Fig. 5), which was owing to poor pore ordering of NAC-MIP ${ }^{16}$.

Rebinding tests: The ability of the imprinted silica to recognize the template was investigated. Fig. 6 showed the amount of the $\mathrm{N}$-acetylcysteine bound to the imprinted silica and blank silica according to the same concentration. The imprinted silica exhibited much higher recognition ability than the blank silica, which proved that the target molecules were not simply adsorbed at the surface, but were trapped in the cavities through ionization bonding. The kinetic uptake of $\mathrm{N}$ acetylcysteine by the imprinted silica was measured in order

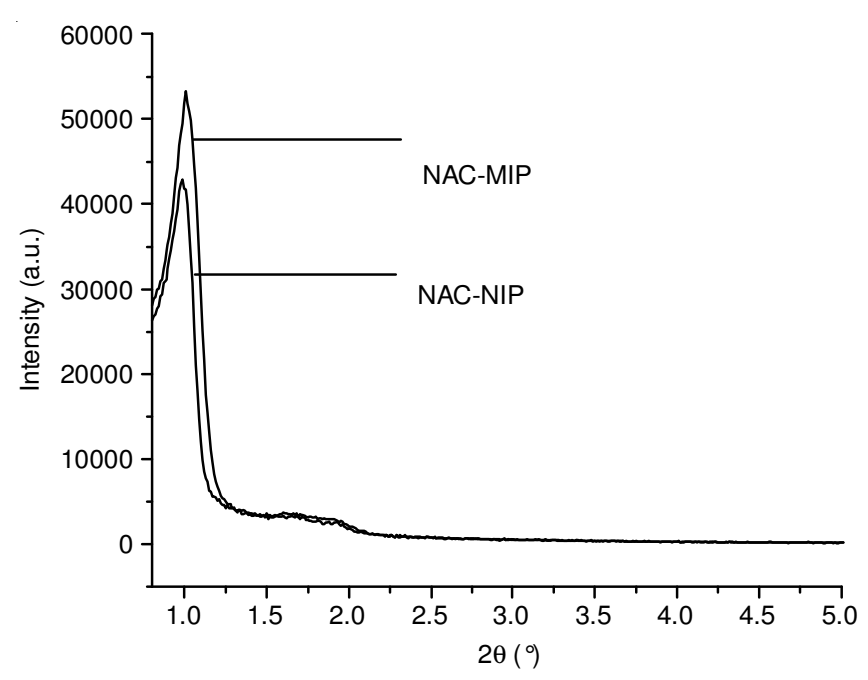

Fig. 5. X-Ray diffractogram of the molecularly imprinted mesoporous silica particles

to evaluate the binding site accessibility (Fig. 6a). An equilibrium amount of $95 \%$ was achieved within 20 min after the addition of $\mathrm{N}$-acetylcysteine and complete equilibrium reached within $30 \mathrm{~min}$. This recognition time was much faster than that of typical molecularly imprinted materials ${ }^{17}$.

The specific recognition ability of the imprinted silica for its structural analogues (HPMA and BPA) was also investigated. The rebinding test was carried out in the same manner. As showed in Fig. 6b, the imprinted silica showed the highest recognition ability for $\mathrm{N}$-acetylcysteine. A partition coefficient $\mathrm{K}$ could be obtained by the following equation: $\mathrm{K}=$ (moles of the analyte bound to silica particles/mass of particles)/(moles of the analyte remaining in the solution/mass of the solution). And the imprinting factor (IF), which was the ratio of partition coefficients of the imprinted and the blank particles (i.e., IF = $\mathrm{K}_{\mathrm{i}} / \mathrm{K}_{\mathrm{b}}$ ), was also calculated. The results (Table-1) indicated that the template molecule, $\mathrm{N}$-acetylcysteine, had the highest partition coefficients and imprinting factors. 3-Hydroxypropyl mercapturic acid had higher and bisphenol-A had the lowest. These results showed that the imprinted silica exhibited highly selective binding affinity for $\mathrm{N}$-acetylcysteine and 3-hydroxypropyl mercapturic acid and demonstrated that the adsorption of these compounds was due to imprinted binding sites and not due to nonspecific binding ${ }^{18}$, while bisphenol-A showed less or no binding. This could be easily explained by their close structural homology to $\mathrm{N}$-acetylcysteine. Fig. $6 \mathrm{~b}$ shows

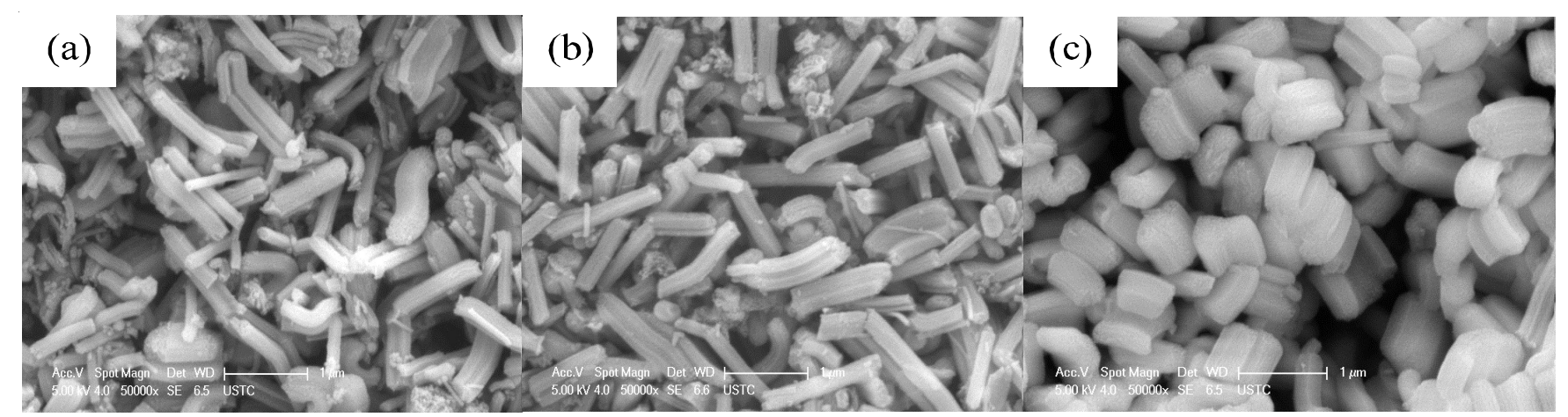

Fig. 4. SEM images of the molecularly imprinted mesoporous silica particles: (a) N-acetylcysteine-MIP (b) N-acetylcysteine-NIP (c) SBA-15 

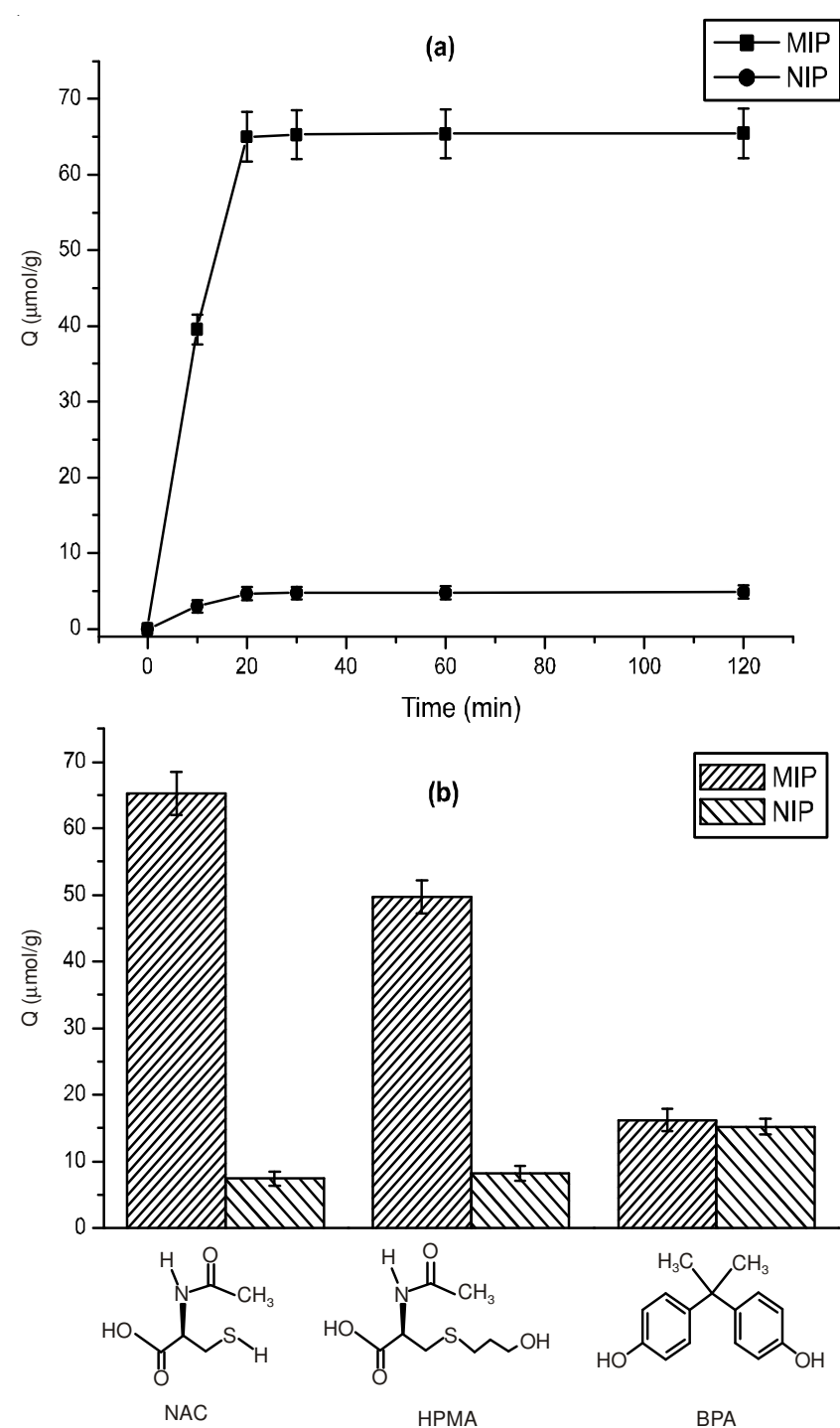

Fig. 6. (a) Kinetic binding profile of $\mathrm{N}$-acetylcysteine to the imprinted silica and the blank silica; (b) Binding selectivity of the imprinted silica and the blank silica

\begin{tabular}{lccc}
\multicolumn{4}{c}{ TABLE-1 } \\
\multicolumn{4}{c}{ PARTITION COEFFICIENTS $\left(\mathrm{K}_{\mathrm{i}}\right.$ and $\left.\mathrm{K}_{\mathrm{c}}\right)$ AND IMPRINTING } \\
FACTORS (IF) FOR THE IMPRINTED SILICA \\
AND THE BLANK SILICA \\
\hline Analyte & NAC & HPMA & BPA \\
\hline $\mathrm{K}_{\mathrm{i}}$ & 1.36 & 0.98 & 0.35 \\
$\mathrm{~K}_{\mathrm{c}}$ & 0.21 & 0.22 & 0.33 \\
$\mathrm{IF}$ & 6.48 & 4.45 & 1.06 \\
\hline
\end{tabular}

that there are $-\mathrm{COOH}$ and $-\mathrm{NH}$ functional groups in the structure of $\mathrm{N}$-acetylcysteine and 3-hydroxypropyl mercapturic acid, the only difference is only a _ $\mathrm{OH}$ instead of -SH in the same position. However, the same groups can't be found in the structural of bisphenol-A. Therefore, the imprinted mesoporous silica particles could be proved to be a powerful material for the selective enrichment of $\mathrm{N}$-acetylcysteine and 3-hydroxypropyl mercapturic acid.

\section{Conclusion}

We have demonstrated the successful molecular imprinting of N-acetylcysteine into SBA-15-type mesoporous organosilica using a surface molecular imprinting technology. The ordered structure of the mesoporous silica was confirmed by scanning electron microscopy and XRD. The infrared and ${ }^{13} \mathrm{C}$ NMR spectroscopy of the polymer further indicated that there were some functional groups in the molecular imprinted polymer which could interact on the template. As expected, the imprinted mesoporous silica particles showed fast kinetic binding for the template and its structural analog due to their thin wall thickness and high surface area. It is believed that this study would enlarge the application of the imprinted mesoporous materials.

\section{ACKNOWLEDGEMENTS}

This work was financially supported by the fundamental research fund for the Central Universities (WK2061020001).

\section{REFERENCES}

1. G. Eisenbrand, M. Otteneder and W. Tang, Toxicology, 190, 249 (2003).

2. K. Karthikeyan, G.T. Arularasu, R. Ramadhas and K.C. Pillai, J. Pharm. Biomed. Anal., 54, 850 (2011).

3. G. Wulff, Chem. Rev., 102, 1 (2002).

4. D. Zhang, Y. Lv, R. Chen and C. Shi, Asian J. Chem., 25, 3922 (2013).

5. C.H. Lu, W.H. Zhou, B. Han, H.H. Yang, X. Chen and X.R. Wang, Anal. Chem., 79, 5457 (2007).

6. D.M. Gao, Z.P. Zhang, M.H. Wu, C.G. Xie, G.J. Guan and D.P. Wang, J. Am. Chem. Soc., 129, 7859 (2007).

7. D. Tian, G.P. Yong, H.W. Tong and S.M. Liu, Chin. J. Chem. Phys., 23, 479 (2010)

8. W.G. Borghard, D.C. Calabro, F.P. DiSanzo, M.M. Disko, J.W. Diehl, J.C. Fried, M.A. Markowitz, M. Zeinali, B.J. Melde and A.E. Riley, Langmuir, 25, 12661 (2009).

9. C.X. Song, X.L. Zhang, C.Y. Jia, P. Zhou, X. Quan and C. Duan, Talanta, 81, 643 (2010).

10. Y. Wang, Y. Yang, L. Xu and J. Zhang, Electrochim. Acta, 56, 2105 (2011).

11. Y. Liu, Z.C. Liu, J. Gao, J.D. Dai, J. Han, Y. Wang, J.M. Xie and Y.S. Yan, J. Hazard. Mater., 186, 197 (2011).

12. D.Y. Zhao, J.L. Feng, Q.S. Huo, N. Melosh, G.H. Fredrickson, B.F. Chmelka and G.D. Stucky, Science, 279, 548 (1998).

13. S. Zheng, L. Gao and J. Guo, J. Solid State Chem., 152, 447 (2000).

14. R. Ojani, E. Ahmadi, J.B. Raoof and F. Mohamadnia, J. Electroanal. Chem., 626, 23 (2009).

15. J.E. Lofgreen, I.L. Moudrakovski and G.A. Ozin, ACS Nano, 5, 2277 (2011).

16. B.M. Jung, M.S. Kim, W.J. Kim and J.Y. Chang, Chem. Commun., 46, 3699 (2010).

17. Y. Du, J. Yang, W. Tang, X.L. Zhu, L. Zhang and Y. Gao, Asian J. Chem., 24, 1175 (2012).

18. L. Chen, S. Xu and J. Li, Chem. Soc. Rev., 40, 2922 (2011). 